\title{
Nedaplatin and irinotecan combination therapy is equally effective and less toxic than cisplatin and irinotecan for patients with primary clear cell adenocarcinoma of the ovary and recurrent ovarian carcinoma
}

\author{
SHIZUO MACHIDA, TOMOMI SATO, HIROYUKI FUJIWARA, YASUSHI SAGA, YUJI TAKEI, \\ AKIYO TANEICHI, HIROAKI NONAKA and MITSUAKI SUZUKI
}

Department of Obstetrics and Gynecology, Jichi Medical University, Tochigi 329-0498, Japan

Received January 12, 2012; Accepted June 15, 2012

DOI: 10.3892/ol.2012.853

\begin{abstract}
This study retrospectively compared nedaplatin and irinotecan hydrochloride (NDP/CPT) combination therapy with cisplatin and irinotecan hydrochloride therapy (CDDP/ CPT) for efficacy and adverse events in the treatment of clear cell adenocarcinoma of the ovary (CCC) and recurrent ovarian carcinoma. A total of 115 patients were included in the present study. NDP/CPT was administered intravenously every 4 weeks (NDP, $60 \mathrm{mg} / \mathrm{m}^{2}$ on day 1; CPT, $50 \mathrm{mg} / \mathrm{m}^{2}$ on days $1,8$ and 15$)$. CDDP/CPT was also administered intravenously (CDDP, $60 \mathrm{mg} / \mathrm{m}^{2}$ on day 1 ; CPT, $60 \mathrm{mg} / \mathrm{m}^{2}$ on days 1,8 and 15). Patients with primary CCC were treated with NDP/CPT in 29 cases and CDDP/CPT in 20 cases. Patients with recurrent ovarian carcinoma were treated with NDP/CPT and CDDP/ $\mathrm{CPT}$ in 33 cases each. No significant difference was observed in the 5-year overall survival (OS)/progression-free survival (PFS) of patients with primary CCC, with the exception of those patients with stages Ia and Ic(b) who underwent NDP/CPT and CDDP/CPT treatments (OS: $58 \%$, PFS: $40 \%$ and OS: $53 \%$ and PFS: $47 \%$, respectively). No significant differences were found in the response rates to NDP/CPT and CDDP/CPT in patients with recurrent ovarian carcinoma (27 and 18\%, respectively). Similarly, there were no significant differences in the 5-year OS and PFS of patients with recurrent ovarian carcinoma treated with NDP/CPT or CDDP/CPT (OS: 15\%, PFS: $3 \%$ and OS: $18 \%$, PFS: $6 \%$, respectively). In terms of the hematological toxicity of grade 3 or above and non-hematological toxicity of grade 2 or above in patients treated with NDP/CPT and CDDP/ CPT, respectively, neutropenia was 23 and 56\%; anemia, 1,
\end{abstract}

Correspondence to: Dr Mitsuaki Suzuki, Department of Obstetrics and Gynecology, Jichi Medical University, 3311-1, Yakushiji, Shimotsuke, Tochigi 329-0498, Japan

E-mail: msuzuki@jichi.ac.jp

Key words: ovarian cancer, cisplatin, nedaplatin, irinotecan hydrochloride, clear cell carcinoma, recurrence and 20\%; thrombocytopenia, 0 and 5\%; nausea, 20 and 52\%; diarrhea, 14 and 25\%; and fever, 2 and 11\%. Accordingly, NDP/ CPT indicated mild toxicity, and was therefore equally effective and less toxic than CDDP/CPT in the treatment of primary $\mathrm{CCC}$ and recurrent ovarian carcinoma.

\section{Introduction}

Clear cell adenocarcinoma of the ovary (CCC) is defined by the World Health Organization as lesions characterized by clear cells growing in solid/tubular or glandular patterns as well as hobnail cells (1). The frequency of CCC in female individuals of Western countries is $8 \%$ (2). The frequency is higher in Japan at 15\% (3). The standard first-line chemotherapy for ovarian epithelial cancer is paclitaxel-platinum combination chemotherapy (T/platinum), which has a response rate of $78 \%$ (4). However, for the treatment of CCC, the response rate is only $22-56 \%(5,6)$. As such, CCC patients have a poorer prognosis than patients with serous cystadenocarcinoma of the ovary (3). Alternative regimens for the treatment of CCC using combinations of camptothecin derivates, irinotecan hydrochloride and cisplatin (CDDP/CPT) have been investigated (7-9). $\mathrm{CDDP} / \mathrm{CPT}$ has also been used as an alternative regimen for the treatment of recurrent ovarian carcinoma $(10,11)$. CDDP/ CPT is regarded as one of the common treatments of primary $\mathrm{CCC}$ and recurrent ovarian carcinoma.

Nedaplatin (cis-diammine glycolato platinum, NDP) is an analog of cisplatin, that shows lower rates of nephrotoxicity and nausea than CDDP. Cervical carcinoma is frequently treated with nedaplatin and irinotecan hydrochloride combination therapy (NDP/CPT). A previous multi-center dose-escalation study was performed to identify appropriate doses of NDP/ CPT for cervical carcinoma patients (12). NDP/CPT was found to have a shorter infusion time and lead to a shorter hospital stay than CDDP/CPT.

NDP has been reported to be effective as a single agent or as combination therapy for the treatment of ovarian carcinoma $(13,14)$, and its basic and clinical efficacy in the treatment of CCC has been demonstrated $(15,16)$. Thus, based on our experience, we applied the postoperative chemotherapy regimen for 
primary CCC from CDDP/CPT to NDP/CPT. This treatment is also currently used for cases of recurrent ovarian carcinoma.

In this study, we retrospectively compared the efficacy and toxicity of NDP/CPT to that of CDDP/CPT in the treatment of $\mathrm{CCC}$ and recurrent ovarian carcinoma.

\section{Patients and methods}

Patient characteristics. In total, 115 patients were included in this study, including patients with primary CCC and recurrent ovarian carcinoma were administered CDDP/CPT or NDP/ CPT at the Jichi Medical University Hospital, Japan. Subjects had no other known co-morbidities. Informed consent was obtained from all patients.

Inclusion criteria consisted of the following: i) age, $\leq 75$ years; ii) Eastern Cooperative Oncology Group performance status, $0-2$; and iii) white blood cell count, $\geq 3,000 / \mu 1$; neutrophil count, $\geq 1,500 / \mu \mathrm{l}$; platelet count, $\geq 10 \times 10^{4} / \mu \mathrm{l}$; hemoglobin level, $\geq 9.0 \mathrm{~g} / \mathrm{dl}$; aspartate aminotransferase and alanine aminotransferase levels, $\leq 3$-fold than the upper limit of the normal value; total bilirubin, $\leq 2.0 \mathrm{mg} / \mathrm{dl}$; urea nitrogen level, $\leq 25 \mathrm{mg} / \mathrm{dl}$; serum creatinine level, $\leq 1.5 \mathrm{mg} / \mathrm{dl}$; and creatinine clearance, $\geq 50 \mathrm{ml} / \mathrm{min}$.

Methods. The NDP/CPT regimen was administered as follows: on day $1,50 \mathrm{mg} / \mathrm{m}^{2}$ of CPT was administered intravenously for $90 \mathrm{~min}$, followed by $60 \mathrm{mg} / \mathrm{m}^{2}$ of NDP for $60 \mathrm{~min}$. On days 8 and 15, patients were administered CPT using the same procedure. Patients were hydrated with 1,000 $\mathrm{ml}$ of electrolyte fluids on day 1 only. This was considered as one course and was repeated every four weeks. The CDDP/CPT regimen was administered as follows: CPT at $60 \mathrm{mg} / \mathrm{m}^{2}$ was administered intravenously for $90 \mathrm{~min}$, followed by CDDP at $60 \mathrm{mg} / \mathrm{m}^{2}$ for $180 \mathrm{~min}$. On day 8 and day 15, CPT only was administered using the same procedure. On days 0-4, patients were hydrated with $2,000 \mathrm{ml}$ of electrolyte fluids. This was considered as one course and was repeated every four weeks.

Granulocyte-colony stimulating factor was administered for patients with grade 4 neutropenia or grade 3 neutropenia with infection. Granisetron hydrochloride (3 $\mathrm{mg})$, a $5-\mathrm{HT}_{3}$ receptor antagonist, was administered intravenously on days 1, 8 and 15 as a prophylactic antiemetic treatment. Loperamide hydrochloride, an anti-diarrheal agent, was orally administered at 1-2 mg/day when required. The subsequent course of chemotherapy was administered when the following conditions were met: white blood cell count, $\geq 3,000 / \mu 1$; neutrophil count, $\geq 1,500 / \mu 1$; platelet count, $\geq 10 \times 10^{4} / \mu 1$; and absence of diarrhea.

The response to treatment was evaluated by computed tomography (CT) images every 2 cycles of chemotherapy in patients with measurable disease. Tumor response was evaluated according to World Health Organization criteria (1979). A complete response (CR) was defined as the disappearance of all clinical and radiological evidence of the tumor for at least four weeks. A partial response (PR) was defined as a decrease of $\geq 50 \%$ in the sum of the products of the longest perpendicular diameters of all measurable lesions for at least four weeks. Progressive disease (PD) was defined as an increase of $>25 \%$ in the sum of the products of the perpendicular diameter of all measurable lesions or the appearance
Table I. Characteristics of patients with primary CCC.

\begin{tabular}{lcc}
\hline & $\begin{array}{c}\text { NDP/CPT } \\
(\mathrm{n}=29)\end{array}$ & $\begin{array}{c}\text { CDDP/CPT } \\
(\mathrm{n}=20)\end{array}$ \\
\hline Age (years) & & \\
Median & 54 & 53.5 \\
Range & $31-69$ & $37-67$ \\
FIGO stage & & \\
Ia/Ic(b) & 14 & 1 \\
Ic(1)/(2)/(a) & 5 & 7 \\
II & 4 & 3 \\
III & 4 & 6 \\
IV & 2 & 3 \\
Residual tumor size $(\mathrm{cm})$ & & \\
0 & 27 & 2 \\
$<1$ & 2 & 2 \\
$>1$ & 0 & \\
\hline
\end{tabular}

CCC, clear cell carcinoma; NDP/CPT, nedaplatin plus irinotecan; $\mathrm{CDDP} / \mathrm{CPT}$, cisplatin plus irinotecan.

of new lesions. Any other events were considered to indicate no change (NC). Progression-free survival (PFS) was defined as the interval from the date of the first chemotherapy administration until the date of recurrence or tumor progression. Overall survival (OS) was defined as the time from the date of chemotherapy until death or the date of the last follow-up contact. Adverse events were graded according to the National Cancer Institute's Common Terminology Criteria for Adverse Events (NCI-CTAE) version 3.0.

Statistical analysis. The Kaplan-Meier method was used to calculate the distribution of patient survival, and its significance in each group was tested using the log-rank test. The $\chi^{2}$ test was used for statistical analysis. $\mathrm{P}<0.05$ was considered statistically significant.

\section{Results}

Primary $C C C$. In the present study, 29 patients with primary CCC were treated using NDP/CPT, and 20 patients were treated using CDDP/CPT (Table I). The median age was 54 years in the NDP/CPT group and 53.5 years in the CDDP/CPT group. According to the International Federation of Gynecology and Obstetrics staging criteria, there were 14 cases with stage Ia/ Ic(b) in the NDP/CPT group and only one such case in the CDDP/CPT group. The reason for this observation may be the change in the indication for postoperative adjuvant chemotherapy during the period involved. During the period of CDDP/CPT use, patients with stage Ic(b) did not undergo chemotherapy, whereas during the period of NDP/CPT use, patients with stage Ic(b) underwent chemotherapy. Patients with stage Ia do not usually receive adjuvant therapy; however, if a residual tumor caused by adhesion is suspected, adjuvant therapy is used. There was no significant difference in the degree of completion of surgery. 


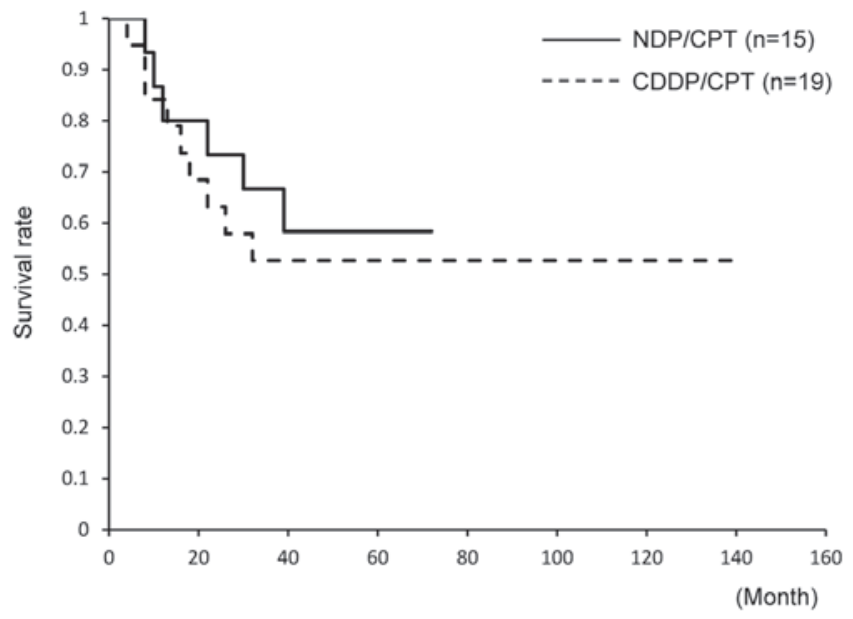

Figure 1. Overall survival (OS) of patients with primary CCC, excluding those with stages Ia and Ic(b). The 5-year OS is 58\% in patients treated with NDP/CPT and 53\% in those treated with CDDP/CPT. No significant difference was observed.

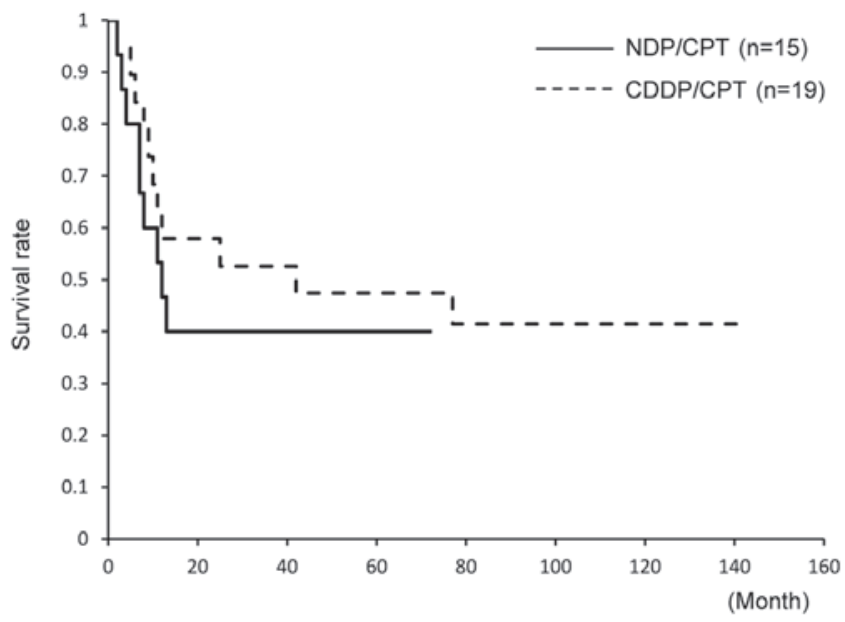

Figure 2. Progression-free survival (PFS) of patients with primary CCC, excluding those with stages Ia and Ic(b). The 5-year PFS is $40 \%$ in patients treated with NDP/CPT and $47 \%$ in those treated with CDDP/CPT. No significant difference was observed.

Survival was assessed by excluding patients with stage Ia/ Ic(b). There was no significant difference in the 5-year OS between the groups; OS was $58 \%$ in patients treated with NDP/CPT and 53\% in those treated with CDDP/CPT (Fig. 1). Similarly, the 5-year PFS did not significantly differ between the groups; PFS was $40 \%$ in patients treated with NDP/CPT and $47 \%$ in those treated with CDDP/CPT (Fig. 2).

Recurrent ovarian carcinoma. Sixty-six patients with recurrent ovarian carcinoma were treated with either NDP/CPT or CDDP/CPT (Table II). The median age was 56 years in the $\mathrm{NDP} / \mathrm{CPT}$ group and 52 years in the CDDP/CPT group. In the two groups, there was a large proportion of patients with stages III-IV, and the most common histological type observed was serous adenocarcinoma. In the two groups, previous treatments mostly consisted of 1 or 2 regimens, and the groups did not show a significant difference. The previous regimen
Table II. Characteristics of patients with recurrent ovarian carcinoma.

\begin{tabular}{|c|c|c|}
\hline & $\begin{array}{c}\mathrm{NDP} / \mathrm{CPT} \\
(\mathrm{n}=33)\end{array}$ & $\begin{array}{c}\mathrm{CDDP} / \mathrm{CPT} \\
(\mathrm{n}=33)\end{array}$ \\
\hline \multicolumn{3}{|l|}{ Age (years) } \\
\hline Median & 56 & 52 \\
\hline Range & $20-72$ & $34-72$ \\
\hline \multicolumn{3}{|l|}{ FIGO stage } \\
\hline I & 5 & 5 \\
\hline II & 1 & 3 \\
\hline III & 23 & 15 \\
\hline IV & 4 & 10 \\
\hline \multicolumn{3}{|c|}{ Histological subtype } \\
\hline Serous & 19 & 18 \\
\hline Clear & 4 & 5 \\
\hline Endometrioid & 5 & 3 \\
\hline Mucinous & 2 & 3 \\
\hline Others & 3 & 4 \\
\hline \multicolumn{3}{|c|}{$\begin{array}{l}\text { Number of } \\
\text { prior chemotherapy } \\
\text { regimens }\end{array}$} \\
\hline 0 & 3 & 2 \\
\hline 1 & 20 & 19 \\
\hline 2 & 9 & 10 \\
\hline 3 & 1 & 2 \\
\hline \multicolumn{3}{|c|}{$\begin{array}{l}\text { Prior chemotherapy } \\
\text { cycle (duplicated) }\end{array}$} \\
\hline $\mathrm{TC}$ & 24 & 13 \\
\hline $\mathrm{JP}$ & 1 & 16 \\
\hline Weekly T & 8 & 5 \\
\hline $\mathrm{DC}$ & 5 & 0 \\
\hline Other & 3 & 8 \\
\hline \multicolumn{3}{|c|}{ Time to recurrence } \\
\hline$<6$ months & 24 & 18 \\
\hline$>6$ months & 9 & 15 \\
\hline
\end{tabular}

NDP/CPT, nedaplatin plus irinotecan; CDDP/CPT, cisplatin plus irinotecan; TC, paclitaxel plus carboplatin; JP, carboplatin plus CDDP; weekly T, weekly paclitaxel; DC, docetaxel plus carboplatin.

most frequently used in the NDP/CPT group was paclitaxel/ carboplatin (TC), whereas it was cisplatin and carboplatin (JP) in the CDDP/CPT group. The NDP/CPT group tended to have a higher 6-month recurrence rate than the $\mathrm{CDDP} / \mathrm{CPT}$ group; however, this difference was not statistically significant.

The NDP/CPT group had a better response rate than the CDDP/CPT group; however, this difference was not statistically significant (27 and 18\%, respectively) (Table III). No significant difference was found in the disease control rate between the two groups (62 and 68\%, respectively).

The 5-year OS was not significantly different $(15$ and $18 \%$, respectively). The median OS was 19 months in the two groups, and it was not significantly different (Fig. 3). The 5-year PFS 
Table III. Treatment outcome.

\begin{tabular}{lccc}
\hline & $\begin{array}{c}\text { NDP/CPT } \\
(\mathrm{n}=33)\end{array}$ & $\begin{array}{c}\text { CDDP/CPT } \\
(\mathrm{n}=33)\end{array}$ & $\mathrm{p}$ \\
\hline $\mathrm{CR}$ & 3 & 3 & \\
$\mathrm{PR}$ & 4 & 1 & \\
$\mathrm{NC}$ & 9 & 11 & \\
$\mathrm{PD}$ & 10 & 7 & \\
$\mathrm{NE}$ & 7 & 11 & \\
$\mathrm{CR}+\mathrm{PR}(\%)$ & $27 \%(7 / 26)$ & $18 \%(4 / 22)$ & $\mathrm{ns}$ \\
$\mathrm{CR}+\mathrm{PR}+\mathrm{NC}(\%)$ & $62 \%(16 / 26)$ & $68 \%(15 / 22)$ & $\mathrm{ns}$ \\
\hline
\end{tabular}

$\mathrm{NDP} / \mathrm{CPT}$, nedaplatin plus irinotecan; CDDP/CPT, cisplatin plus irinotecan; $\mathrm{CR}$, complete response; $\mathrm{PR}$, partial response; $\mathrm{NC}$, no change; $\mathrm{PD}$, progressive disease; NE, not evaluable; ns, not significant.

was $3 \%$ in the NDP/CPT group and $6 \%$ in the CDDP/CPT group; however, no significant difference was found between the two groups. The median PFS also did not show any difference (6 and 4 months, respectively, Fig. 4).

Toxicity. Toxicity was assessed among patients with primary $\mathrm{CCC}$ and recurrent ovarian carcinoma. Toxicity was compared between the 62 patients treated with NDP/CPT and the 53 patients treated with CDDP/CPT (Table IV). For hematotoxicity of grade 3 or above, neutropenia was observed in $23 \%$ of patients treated with NDP/CPT, which was lower than that in patients treated with CDDP/CPT (56\%). Anemia and thrombocytopenia were also lower in patients treated with NDP/CPT. With regards to non-hematotoxicity of grade 2 or above, there was a lower rate of nausea in patients treated with NDP/CPT compared to those treated with CDDP/CPT (20 and $52 \%$, respectively). There were also lower rates of diarrhea and fever in the NDP/CPT group.

\section{Discussion}

The results of this retrospective analysis demonstrate that NDP/CPT treatment was equally effective yet less toxic than $\mathrm{CDDP} / \mathrm{CPT}$ in the treatment of primary $\mathrm{CCC}$ and recurrent ovarian carcinoma.

NDP is an analog of cisplatin that has the same amine carrier ligands as cisplatin but has a different leaving group, a 5-membered ring structure in which glycolate is bound to the platinum ion as a bidentate ligand. This product is approximately 10 times more water-soluble than cisplatin and, unlike cisplatin, shows limited binding to plasma proteins. As such, it causes less renal, gastrointestinal, and neural toxicity, and there is no need for hydration during its administration (17).

In an in vitro study of chemosensitivity, an MTT assay of the ovarian cancer cell strain 65 showed that the inhibition rate of the NDP tumor was $62 \%$ and that of CDDP was $55 \%$. For clear cell adenocarcinoma, the inhibition rate of the NDP tumor was $50 \%$ and that of CDDP was $37 \%$ (15). In a phase II clinical study, patients with ovarian carcinoma who were administered a dose of $100 \mathrm{mg} / \mathrm{m}^{2}$ of NDP every 4 weeks

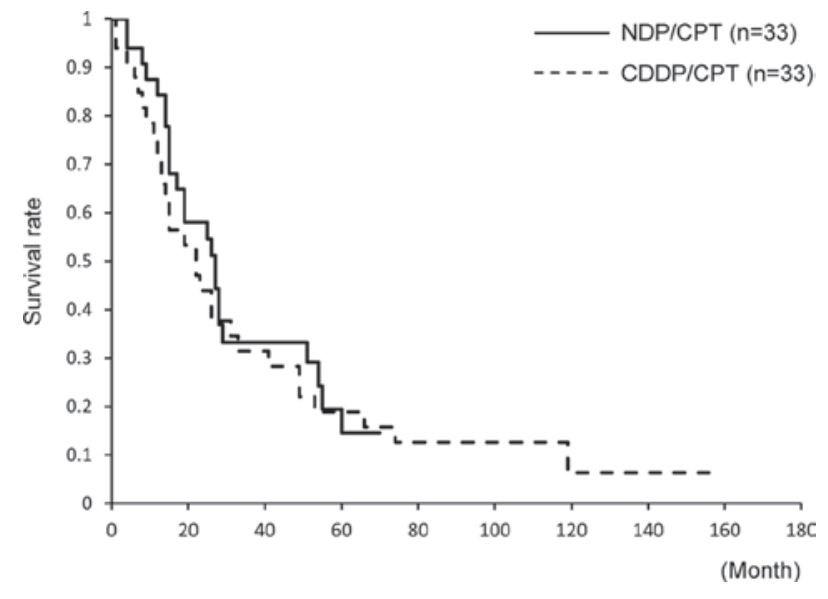

Figure 3. Overall survival (OS) of recurrent ovarian carcinoma. The 5-year OS is $15 \%$ in patients treated with NDP/CPT and $18 \%$ in patients treated with CDDP/CPT. No significant difference is observed. The median OS in the two groups is 19 months. No significant difference was observed.

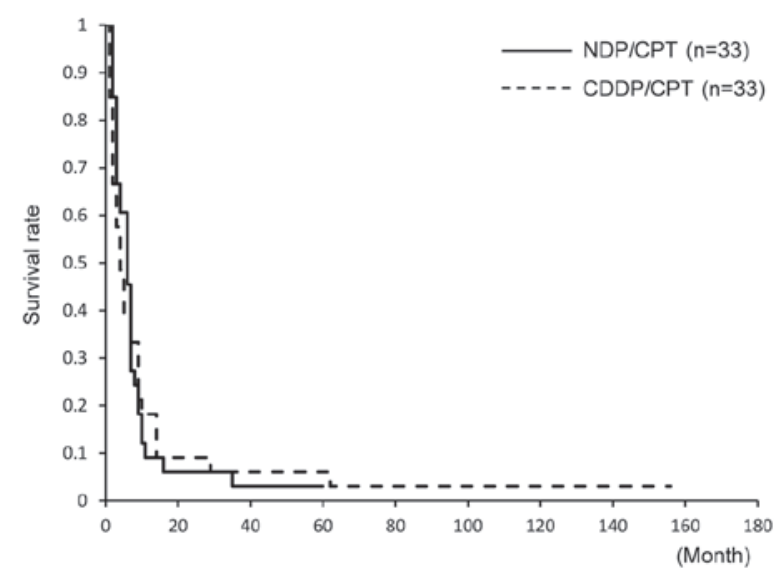

Figure 4. Progression-free survival (PFS) of recurrent ovarian carcinoma The 5-year PFS is $3 \%$ in patients treated with NDP/CPT and $6 \%$ in patients treated with CDDP/CPT. No significant difference was observed. The median PFS is 6 months in patients treated with NDP/CPT and 4 months in patients treated with CDDP/CPT. No significant difference was observed.

showed a response rate of $37.7 \%$ (23/61). The response rate was $35.7 \%(15 / 42)$ in patients with a previous history of adjuvant therapy, whereas the response rate was $33 \%$ in patients with clear cell adenocarcinoma. This study concluded that NDP was equally effective and less toxic than CDDP for the treatment of ovarian carcinoma (13).

CPT is a derivate of camptothecin, and it inhibits topoisomerase I. It may be used either as a single agent or in combination therapy for gastric, colon, lung and cervical carcinomas (18). As a single agent, CPT had a response rate of $20-25 \%$ in cases of recurrent or refractory ovarian carcinoma (10).

NDP/CPT has been used as chemotherapy for lung, cervical, and testicular carcinomas $(12,19-21)$. In a phase I study, the Japanese Gynecologic Oncology Group (JGOG) established a recommended dose for patients with cervical carcinoma patients. A phase II study investigating its use as postoperative adjuvant chemotherapy for cervical carcinoma is currently being performed (19). Few available reports 
Table IV. Adverse events.

A, Hematological toxicity.

\begin{tabular}{|c|c|c|c|c|c|c|c|c|c|c|c|}
\hline & \multicolumn{5}{|c|}{ NDP/CPT } & \multicolumn{6}{|c|}{ CDDP/CPT } \\
\hline & \multicolumn{4}{|c|}{$\mathrm{n}=124$ courses } & \multirow{2}{*}{$\begin{array}{l}\mathrm{G} 3 / 4 \\
(\%)\end{array}$} & \multicolumn{4}{|c|}{$\mathrm{n}=106$ courses } & \multirow{2}{*}{$\begin{array}{c}\mathrm{G} 3 / 4 \\
(\%)\end{array}$} & \multirow[b]{2}{*}{$\mathrm{p}^{\mathrm{a}}$} \\
\hline & G1 & G2 & G3 & G4 & & G1 & G2 & G3 & G4 & & \\
\hline Leukopenia & 29 & 51 & 5 & 0 & 4 & 21 & 52 & 24 & 7 & 29 & $<0.01$ \\
\hline Neutropenia & 16 & 38 & 24 & 5 & 23 & 17 & 28 & 35 & 24 & 56 & $<0.01$ \\
\hline Anemia & 26 & 14 & 1 & 0 & 1 & 40 & 37 & 17 & 4 & 20 & $<0.01$ \\
\hline Thrombocytopenia & 2 & 0 & 0 & 0 & 0 & 21 & 7 & 4 & 1 & 5 & $<0.05$ \\
\hline
\end{tabular}

B, Non-hematological toxicity.

\begin{tabular}{|c|c|c|c|c|c|c|c|c|c|c|c|}
\hline & \multicolumn{4}{|c|}{$\mathrm{n}=124$ courses } & \multirow{2}{*}{$\begin{array}{c}\mathrm{G} 2 / 3 / 4 \\
(\%)\end{array}$} & \multicolumn{4}{|c|}{$\mathrm{n}=106$ courses } & \multirow{2}{*}{$\begin{array}{c}\mathrm{G} 2 / 3 / 4 \\
(\%)\end{array}$} & \multirow[b]{2}{*}{$\mathrm{p}^{\mathrm{a}}$} \\
\hline & G1 & G2 & G3 & G4 & & G1 & $\mathrm{G} 2$ & G3 & G4 & & \\
\hline Nausea & 54 & 24 & 1 & 0 & 20 & 35 & 43 & 12 & 0 & 52 & $<0.01$ \\
\hline Diarrhea & 29 & 15 & 2 & 0 & 14 & 13 & 16 & 9 & 1 & 25 & $<0.05$ \\
\hline Hepatotoxicity & 23 & 1 & 1 & 0 & 2 & 6 & 2 & 1 & 0 & 3 & ns \\
\hline Nephrotoxicity & 2 & 0 & 0 & 0 & 0 & 1 & 0 & 0 & 0 & 0 & $\mathrm{~ns}$ \\
\hline Fervescence & 2 & 2 & 0 & 0 & 2 & 1 & 12 & 0 & 0 & 11 & $<0.01$ \\
\hline
\end{tabular}

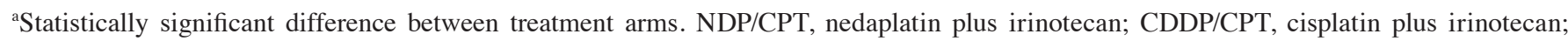
G, grade; ns, not significant.

regarding the use of NDP/CPT for ovarian carcinoma are available; however, one study showed CR to NDP/CPT in patients with CCC metastasis to the common iliac lymph node (16). It was also reported that platinum/CPT therapy, including CDDP/CPT and NDP/CPT, was effective in 31 patients with ovarian carcinoma (14).

The doses of NDP and CPT are determined by body surface area; however, this method may be improved in the future. For instance, Ishibashi et al developed Ishibashi's formula to calculate the dose of NDP on the basis of renal function as with carboplatin (CBDCA) (22). The correlation between the predicted area under the curve (AUC) and observed AUC values suggested by Ishibashi's formula was confirmed from a study in which we participated (23). In the future, Ishibashi's formula may be used to determine the appropriate dose of NDP. SN-38, an active metabolite of $\mathrm{CPT}$, is detoxified by glucuronidation with uridine diphosphate gluconosyltransferase (UGT)1A1. The homozygotes and double heterozygotes of UGT1A1* 6 and *28 (*6/:6, *28/*28, *6/"28) were significantly associated with severe neutropenia (24). However, the necessity of the dose adjustment of CPT on the basis of the UGT1A1 polymorphism has yet to be determined (25).

Sugiyama et al observed that CDDP/CPT treatment had a response rate of $40 \%$ in patients with recurrent or refrac- tory ovarian carcinoma (11). Gershenson et al reported that platinum-sensitive and non-sensitive ovarian carcinomas had response rates of 75 and $33 \%$, respectively (10). Recently, single-agent chemotherapy has been recommended for cases of platinum-resistant ovarian carcinoma. Platinum-based combination chemotherapy is recommended for platinum-sensitive recurrent ovarian carcinoma (Ovarian Cancer Guideline, http:// www.nccn.org/professionals/physician_gls/f_guidelines.asp). Paclitaxel/carboplatin (TC) is often used for platinum-sensitive recurrent ovarian carcinoma. Consequently, the applicability of platinum/CPT is limited for recurrent cases. For patients with platinum-sensitive recurrent ovarian carcinoma that cannot tolerate paclitaxel due to numbness and allergy, NDP/CPT is an alternative chemotherapeutic agent.

Findings of recent studies have suggested that CDDP/CPT has an efficacy similar to or better than T/platinum in patients with primary CCC. In a retrospective study that compared 46 cases of CDDP/CPT and 126 cases of T/platinum with optimal debulking in stages II-IV, the 2-year PFS for CDDP/ CPT was $86 \%$, which was higher than that of T/platinum (44\%) (7). In another retrospective study of 82 patients treated with TC and 35 patients treated with CDDP/CPT, equal efficacy was observed between the groups (8). In the JGOG's randomized phase II trial, a comparison between 48 cases treated with 
CDDP/CPT and 50 cases treated with TC, the treatments were equally tolerated, and there was no significant difference in PFS. Since there were numerous patients in the CDDP/CPT group with large residual tumor cells, a sub-analysis was performed in those with $2-\mathrm{cm}$ residual tumors. In this sub-analysis, the PFS tended to be longer in the CDDP/CPT group, although the difference was not statistically significant $(\mathrm{p}=0.056)(9)$.

At present, the JGOG and Gynecologic Cancer Intergroup (GCIG) are participating in an international cooperative randomized phase III trial (GCIG/JGOG3017 ovarian trial) to compare CDDP/CPT and TC as initial first-line chemotherapy for the treatment of $\mathrm{CCC}$ (9). If results are obtained that show CDDP/CPT is more suitable than TC for treating patients with CCC, a prospective study should be performed to establish whether a regimen with less toxicity, such as NDP/CPT, is suitable for the treatment of CCC.

\section{References}

1. Serov SF, Scully RE and Sobin LH (eds): International Histologic Classification of Tumors, No. 9. Histological Typing of Ovarian Tumors. World Health Organization, Geneva, 1973.

2. Heintz AP, Odicino F, Maisonneuve $\mathrm{P}$, et al: Carcinoma of the ovary. FIGO 6th Annual Report on Results of Treatment in Gynecological Cancer. Int J Gynaecol Obstet 95: S161-S192, 2006.

3. Sugiyama T, Kamura T, Kigawa J, et al: Clinical characteristics of clear cell carcinoma of the ovary: a distinct histologic type with poor prognosis and resistance to platinum-based chemotherapy. Cancer 88: 2584-2589, 2000.

4. Du Bois A, Herrstedt J, Hardy-Bessard AC, et al: Phase III trial of carboplatin plus paclitaxel with or without gemcitabine in first-line treatment of epithelial ovarian cancer. J Clin Oncol 28 : 4162-4169, 2010

5. Utsunomiya H, Akahira J, Tanno S, et al: Paclitaxel-platinum combination chemotherapy for advanced or recurrent ovarian clear cell adenocarcinoma: a multicenter trial. Int J Gynecol Cancer 16: 52-56, 2006.

6. Enomoto T, Kuragaki C, Yamasaki M, et al: Is clear cell carcinoma and mucinous carcinoma of the ovary sensitive to combination chemotherapy with paclitaxel and carboplatin? Proc Am Soc Clin Oncol 22: abs. 1797, 2003.

7. Takano M, Kikuchi Y, Yaegashi N, et al: Adjuvant chemotherapy with irinotecan hydrochloride and cisplatin for clear cell carcinoma of the ovary. Oncol Rep 16: 1301-1306, 2006.

8. Takano M, Sugiyama T, Yaegashi N, et al: Progression-free survival and overall survival of patients with clear cell carcinoma of the ovary treated with paclitaxel-carboplatin or irinotecancisplatin: retrospective analysis. Int J Clin Oncol 12: 256-260, 2007.

9. Takakura S, Takano M, Takahashi F, et al: Randomized phase II trial of paclitaxel plus carboplatin therapy versus irinotecan plus cisplatin therapy as first-line chemotherapy for clear cell adenocarcinoma of the ovary: a JGOG study. Int J Gynecol Cancer 20: $240-247,2010$
10. Gershenson DM: Irinotecan in epithelial ovarian cancer. Oncology (Williston Park) 16: 29-31, 2002.

11. Sugiyama T, Yakushiji M, Nishida T, Ushijima K, Okura N, Kigawa $J$ and Terakawa N: Irinotecan (CPT-11) combined with cisplatin in patients with refractory or recurrent ovarian cancer. Cancer Lett 128: 211-218, 1998.

12. Machida S, Ohwada M, Fujiwara H, et al: Phase I study of combination chemotherapy using irinotecan hydrochloride and nedaplatin for advanced or recurrent cervical cancer. Oncology 65: 102-107, 2003.

13. Kato T, Nishimura H, Yakushiji M, et al: Phase II study of 254-S (cis-diammine glycolato platinum) for gynecological cancer. Gan To Kagaku Ryoho 19: 695-701, 1992.

14. Ota T, Takeshima N and Takizawa K: Second-line chemotherapy for carboplatin/paclitaxel-refractory ovarian cancer: are multiagent chemotherapies of little value truly? Eur J Gynaecol Oncol 32: 471-475, 2011.

15. Koshiyama M, Kinezaki M, Uchida T and Sumitomo M: Chemosensitivity testing of a novel platinum analog, nedaplatin (254-S), in human gynecological carcinomas: a comparison with cisplatin. Anticancer Res 25: 4499-4502, 2005.

16. Nishida M, Tsunoda H, Ichikawa Y and Yoshikawa H: Complete response to irinotecan hydrochloride and nedaplatin in a patient with advanced ovarian clear cell carcinoma. Int J Clin Oncol 9: 403-405, 2004.

17. Sasaki Y, Amano T, Morita M, et al: Phase I study and pharmacological analysis of cis-diammine (glycolato) platinum (254-S; NSC 375101D) administered by 5-day continuous intravenous infusion. Cancer Res 51: 1472-1477, 1991.

18. Rosen LS: Irinotecan in lymphoma, leukemia, and breast, pancreatic, ovarian, and small-cell lung cancers. Oncology (Williston Park) 12: 103-109, 1998.

19. Yamamoto K, Kokawa K, Umesaki N, et al: Phase I study of combination chemotherapy with irinotecan hydrochloride and nedaplatin for cervical squamous cell carcinoma: Japanese gynecologic oncology group study. Oncol Rep 21: 1005-1009, 2009.

20. Oshita F, Ohe M, Honda T, et al: Phase II study of nedaplatin and irinotecan with concurrent thoracic radiotherapy in patients with locally advanced non-small-cell lung cancer. Br J Cancer 103: 1325-1323, 2010.

21. Miki T, Nomoto T, Nakagawa S, et al: The salvage chemotherapy for refractory testicular cancer with novel anticancer agents. Hinyokika Kiyo 45: 811-814, 1999.

22. Ishibashi T, Yano Y and Oguma T: A formula for predicting optimal dose of nedaplatin based on renal function in adult cancer patients. Cancer Chemother Pharmacol 50: 230-236, 2002.

23. Sato S, Fujiwara H, Oishi T, et al: Evaluation of a formula for individual dosage of nedaplatin based on renal function. Cancer Chemother Pharmacol 69: 599-603, 2012.

24. Minami H, Sai K, Saeki M, et al: Irinotecan pharmacokinetics/ pharmacodynamics and UGT1A genetic polymorphisms in Japanese: roles of UGT1A1*6 and *28. Pharmacogenet Genomics 17: 497-504, 2007.

25. Toffoli G, Cecchin E, Corona G, et al: The role of UGT1A1*28 polymorphism in the pharmacodynamics and pharmacokinetics of irinotecan in patients with metastatic colorectal cancer. J Clin Oncol 24: 3061-3068, 2006 